\title{
CAPITULO 1 \\ Atención de trastornos hipertensivos durante el embarazo en una empresa social del Estado de la ciudad de Barranquilla*
}

\author{
Attention of hypertensive disorders during pregnancy in a \\ social company of the state of the city of Barranquilla
}

\author{
CAROL BELEÑo AgUDELO ${ }^{1}$ \\ MABEL SALAZAR ARAÚJO² \\ LUIS MERCADO RODRÍGUEZ ${ }^{3}$ \\ ZULEIMA ALTAHONA RODRÍGUEZ ${ }^{4}$
}

\footnotetext{
* Producto de la investigación para la tesis de maestría titulada: "Atención de trastornos hipertensivos durante el embarazo en una empresa social del Estado de la ciudad de Barranquilla (Atlántico)”, Universidad Simón Bolívar.

1 Estudiante del programa Maestría en Auditoría y sistemas de calidad en los servicios de salud. cbeleño@unisimonbolivar.edu.co

2 Estudiante del programa Maestría en Auditoría y sistemas de calidad en los servicios de salud. msalazar@unisimonbolivar.edu.co

3 Estudiante del programa Maestría en Auditoría y sistemas de calidad en los servicios de salud. lrodriguez@unisimonbolivar.edu.co

4 Magíster en Auditoría y sistemas de calidad en los servicios de salud, Universidad Simón Bolívar, enfermera egresada de la misma Universidad.

zaltahona@unisimonbolivar.edu.co.
} 


\title{
RESUMEN
}

Las Empresas Sociales del Estado en Colombia (E.S.E.), son las entidades a través de las cuales los colombianos reciben los servicios de salud por parte de gobierno nacional o las entidades territoriales correspondientes. En este caso, el Hospital Niño Jesús de la ciudad de Barranquilla fue escogido para analizar cuantitativamente el grado de conocimiento que tienen los galenos y personal de apoyo sobre la Guía de Prácticas Clínicas para los problemas asociados a la hipertensión gestacional, que es una publicación expedida por las entidades de salud distritales y nacionales, y que busca la estandarización en los procedimientos en la atención y el manejo a las pacientes con estas patologías.

Este estudio pretende conocer cómo y con qué frecuencia son utilizadas las pautas establecidas en este compendio por parte del personal médico encargado de las áreas de ginecología y obstetricia de este centro de salud. Entre las causas que motivan esta investigación está el hecho de que a pesar de los avances de la ciencia y la tecnología en el transcurrir del tiempo, ciertas enfermedades asociadas a la hipertensión en mujeres en estado de embarazo tales como la preeclampsia, la eclampsia, el síndrome de Hellp; aún siguen manteniéndose como principales determinadores en los decesos de mujeres embarazadas.

Palabras clave: trastornos hipertensivos, gestante, preeclampsia, eclampsia, prácticas clínicas.

\begin{abstract}
The State Social Enterprises in Colombia (E.S.E.) are the entities through which colombians receive health services from the national government or the corresponding territorial entities. In this case, the "Baby Jesus Hospital" of the city of Barranquilla; was chosen to analyze quantitatively the
\end{abstract}


degree of knowledge that the doctors and support personnel have on the Clinical Practice Guidelines for problems associated with gestational hypertension, which is a publication issued by the district and national health entities and seeking standardization in the procedures in the attention and the management to the patients with these pathologies.

This study aims to know how and with what frequency, the guidelines established in this compendium are used by medical personnel in charge of the gynecology and obstetrics areas of this health center. Among the reasons for this research is the fact that in spite of advances in science and technology over time, certain diseases associated with hypertension in pregnant women such as Preeclampsia, Eclampsia, Hellp syndrome; still remain as main determiners in the deaths of certain numbers of pregnant women.

Key words: hypertensive disorders, pregnant woman, preeclampsia, eclampsia, clinical practices.

\section{INTRODUCCIÓN}

Debido a falencias en la administración, organización y manejo de recursos destinados a los rubros de la salud en Colombia, actualmente, algunas enfermedades no disminuyen su cuota de mortalidad periódica, a pesar de que existan planes de mejoramiento, diseñados por los entes administrativos del área de la salud que tratan de controlar las cifras de personas afectadas, mejorar los planes de contingencia, corregir errores que se cometían con anterioridad y llevar a cabo estrategias para erradicarlas o disminuir su presencia en la población (Landázuri, Restrepo, \& Trejos, 2006).

Lo anterior se traduce en que algunos grupos de la población con ciertas características socio-económicas, no tengan acceso a servicios de salud, o si los tiene, no se le prestan de la manera adecuada, siendo esta situación 
una circunstancia que permite ubicarnos en el tema que se trata en este escrito, que es la exclusión o falta de aplicación de las guías de prácticas clínicas para ciertas enfermedades (Gaillard R. et al, 2011)

La falta de conocimientos o de difusión sobre las guías de prácticas clínicas de cualquier enfermedad, siendo este el caso de los trastornos hipertensivos en mujeres gestacionales, ocasiona -entre otras cosas- que el servicio prestado por las instituciones prestadoras de salud (IPS) se maneje de forma independiente y que haya un sinfín de diferencias entre uno y otro, convirtiéndose esto en un motivo para que la efectividad en los resultados no sean siempre efectivos, pues una de las ventajas que se obtienen al difundir estas guías, es que los procedimientos descritos en ellos, tienen sus bases en estudios e investigaciones científicas que garantizan los mejores resultados, y en donde los principales beneficiarios son los usuarios del sistema de salud y en especial en este caso, las mujeres gestantes (LilDommar, Suárez, Rojas, Marcano, \& Noccio, 2009).

A pesar de los esfuerzos del Ministerio de Salud y las instituciones descentralizadas que provienen de este, la cantidad de mujeres embarazadas que presentan enfermedades relacionadas con la hipertensión tales como la eclampsia, preeclampsia y el síndrome de Hellp, siguen presentes en las estadísticas como una de las principales complicaciones que causan muertes maternas y perinatales, junto con otras patologías como las infecciones y las hemorragias (en ambos casos postparto) según datos de MacDonald et al, (2012).

La estandarización de esos procedimientos al interior de las diferentes comunidades, instituciones, hasta ciudades, permitiría aumentar la calidad en el servicio, y los beneficios para la salud en general serían distribuidos según unos criterios justos (Romero \& González, 2011). 


\section{APLICACIÓN DE LAS GUÍAS PRÁCTICAS EN ATENCIÓN} A GESTANTES

Las guías para prácticas clínicas de trastornos hipertensivos gestacionales son compendios emanados por entidades de carácter gubernamental, en la rama de salud o entidades educativas en sus áreas de investigación científica y desarrollo, que contienen observaciones, indicaciones y/o recomendaciones, que llevadas de manera metódica, contienen los saberes y competencias necesarias para que los actores principales de las entidades de salud (médicos, enfermeras, paramédicos) apliquen de una manera adecuada y eficiente, procedimientos de atención a pacientes femeninas en estado de embarazo garantizando así, excelentes resultados y además, logrando unificar en todas las E.S.E. y sus representantes de servicio estos procedimientos en un ámbito regional (Arrieta, Blanquicet, Borero, \& $\mathrm{Mu}$ ñoz, 2015) (Rodríguez et al, 2011).

La aplicación de estas guías en las entidades de salud tiene ciertas ventajas, tal como que sus autores son profesionales en el ramo, tienen una amplia trayectoria académica y clínica en el tema que se está tratando (Ortega, Hernández, Mariano \& Herrera, 2015). Las guías también logran sintetizar un gran volumen de información en formatos sencillos, de fácil comprensión y utilización, facilitan la toma de decisiones al personal involucrado en los casos que hacen referencia al tema médico en mención; el uso de las guías propende a lograr equilibrios entre teoría y práctica, entre actores activos y pasivos de estos escenarios de la salud, y entre experiencias de una u otra persona. Además, se pueden adaptar guías del ámbito nacional para aplicarlas en un entorno local (Kovanci \& Gregg, 2010); (Salas, Velasquez, Villareal \& Arata, 2004).

Estas guías deben ser diseñadas de manera que su contenido sea claro, lógico, conciso, fácil de seguir; no deben presentar desviaciones o tergiversar 
la información que en ella se explica, su contenido debe ser fiable para que cualquier profesional lo pueda entender, debe ser flexible para que pueda aplicarse a cualquier paciente dentro de la diversidad que pueda presentarse, y sus autores deben ser profesionales de diferentes especialidades pero que sean relacionadas con el tema en cuestión (Thompson Williams \& Miller, 2009).

\section{METODOLOGÍA}

Para poder obtener resultados confiables de un análisis que estime el objetivo principal de este tema, que es el saber si los profesionales del E.S.E. Niño Jesús de Barranquilla, conocen la "Guía de prácticas clínicas para trastornos hipertensivos gestacionales", se ha determinado que el método a utilizar para este estudio es de tipo cuantitativo, ya que lo que se busca es analizar a un grupo de individuos que se dividen en forma aleatoria en subgrupos y se analizan con respecto a un factor de medida en el E.S.E. Hospital Niño Jesús de la ciudad de Barranquilla, en el departamento del Atlántico.

Para la determinación del estudio se tomó una muestra de 11 individuos y se aplicó una herramienta de captura de datos, llamada el método estadístico Kudger-Rchardson que usa respuestas dicotómicas para obtener el dato deseado.

\section{RESULTADOS}

Una vez cumplida la etapa de recopilación y análisis de la información, se procede a reunir los datos obtenidos y exponer los factores inherentes al proceso de investigación.

El resultado de este primer acercamiento, nos muestra que el 84,85 \% de la población objeto de análisis de estudio, pertenece al género masculino, mientras el 15,15\% restante, pertenecen al género femenino. 


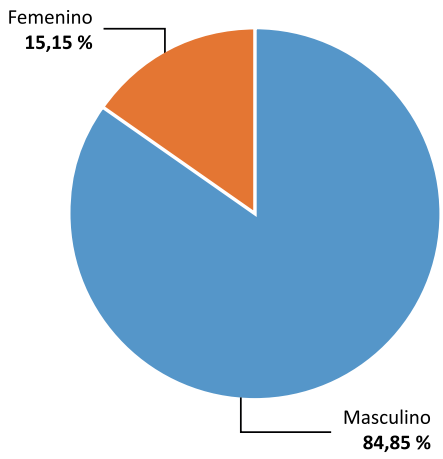

Figura 1

Caracterización de la población

Según los resultados del gráfico, se indica que el 33,33 \% de los entrevistados son médicos de profesión, un 27,27 \% son enfermeras, y otro 27,27 \% son auxiliares de enfermería y el 12,12 \% restante, son médicos especialistas en ginecología.

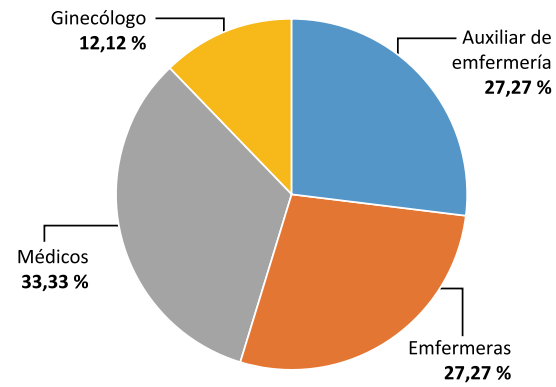

Figura 2

Perfil profesional de la población en estudio

Además, aproximadamente un $85 \%$ en promedio de la población en estudio (enfermeras, auxiliares, médicos y ginecólogos) sí tienen conocimiento de la existencia de las Guías de Prácticas Clínicas, para trastornos hipertensivos gestacionales, mientras que un $10 \%$ aproximadamente, dice no tener conocimiento sobre estas y un $6,25 \%$ promedio, no responde respecto al interrogante. 
Investigaciones y casos del sector salud de la región Caribe

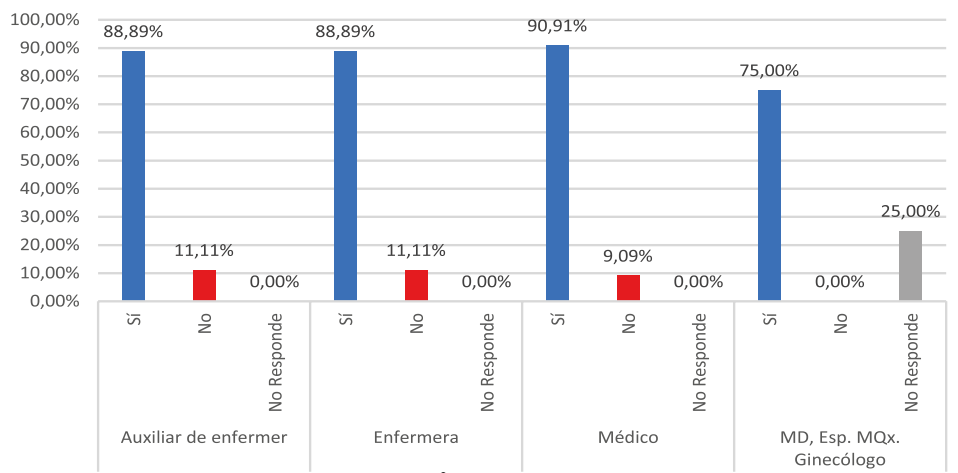

Figura 3

Conocimiento de existencia y aplicación Guías de Prácticas Clínicas

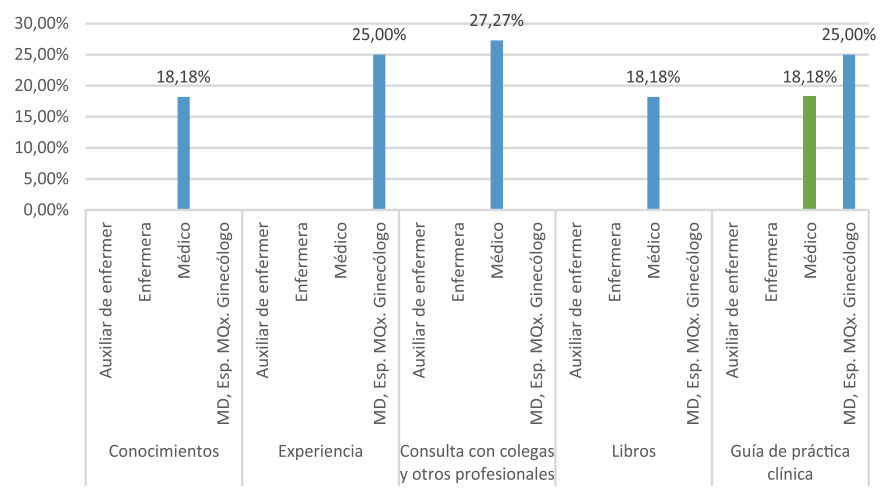

Figura 4

Criterio para toma de decisiones

Según la ilustración, al momento de tomar decisiones, la prioridad la tienen los médicos con un 27,27\%, y lo hacen consultando con colegas y otros profesionales, el segundo lugar lo tienen los médicos especialistas y ginecólogos con un $25 \%$, y lo hacen atendiendo la guía de prácticas clínicas. Le sigue con otro $25 \%$ los médicos especialistas en ginecología, basándose en la experiencia, y compartiendo un 18,18 \% los médicos, toman decisiones basados en sus conocimientos, otro $18,18 \%$ de profesionales de la salud, pertenecientes a esta muestra lo hacen ayudándose con libros. Y por último, un 18,18 \% de médicos lo hacen con la ayuda de la guía práctica clínica. 
Atención de trastornos hipertensivos durante el embarazo en una empresa social del Estado de la ciudad de Barranquilla

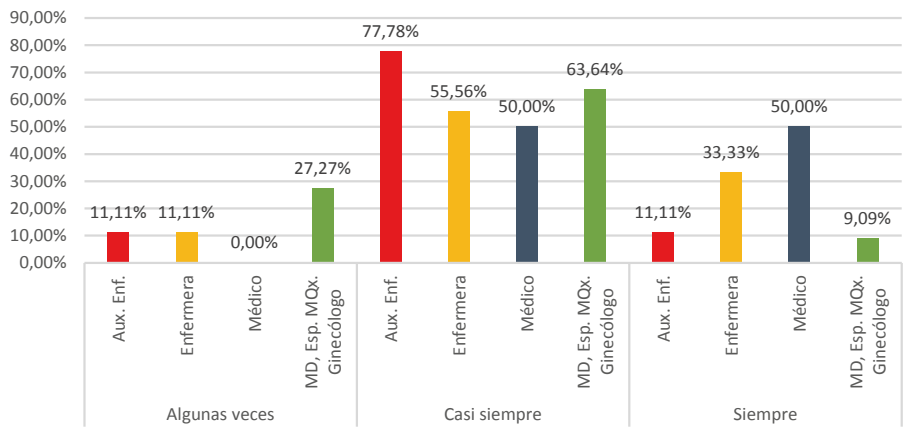

Figura 5

Niveles de adherencia a la Guía práctica de la Clínica

De acuerdo a la encuesta, los especialistas, médicos, enfermeras y auxiliares de enfermería muestran tener con un 61,75 \% “Casi siempre” un buen nivel de adherencia con la guía práctica clínica, seguido de un 25,88 \% que muestra que "Siempre" se ciñen a la guía práctica clínica, y finalmente un 12,37 \% “algunas veces” siguen la guía práctica clínica.

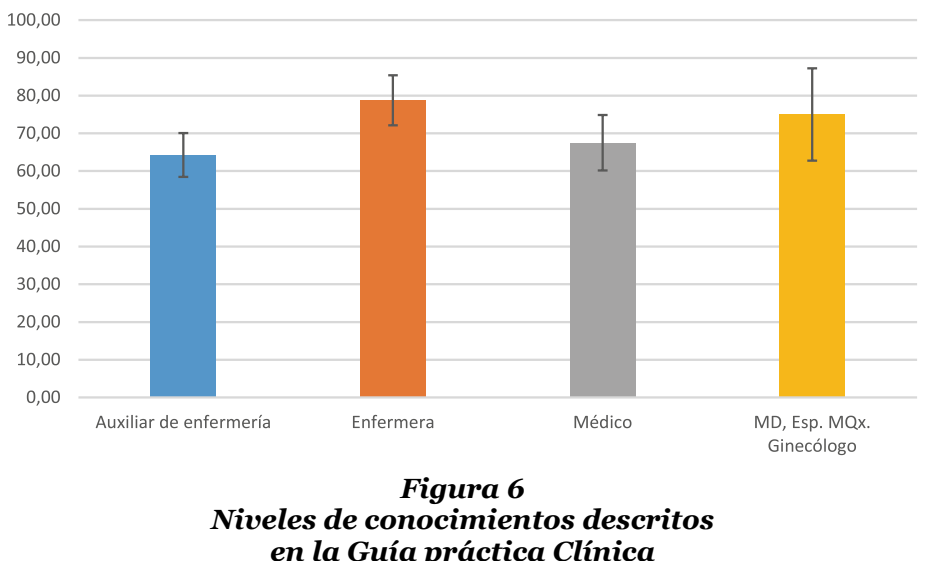

Lo que permite observar este gráfico, es que los niveles de conocimientos de los procedimientos descritos en la Guía práctica clínica, se manejan en su mayoría en un 79 \% por las enfermeras, seguidos de un 74 \% por los mé- 
dicos especialistas en ginecología, el tercer lugar lo tienen los médicos con un $68 \%$ y por último las auxiliares de enfermería con un $64 \%$.

Como se evidencia, un 84,85\% dice que, Sí hace consultas en la guía para prácticas clínicas, seguido de un 12,12 \% que dicen No tener disponible la guía para prácticas clínicas y un 3,03\% dice que no hace consultas a este compendio.

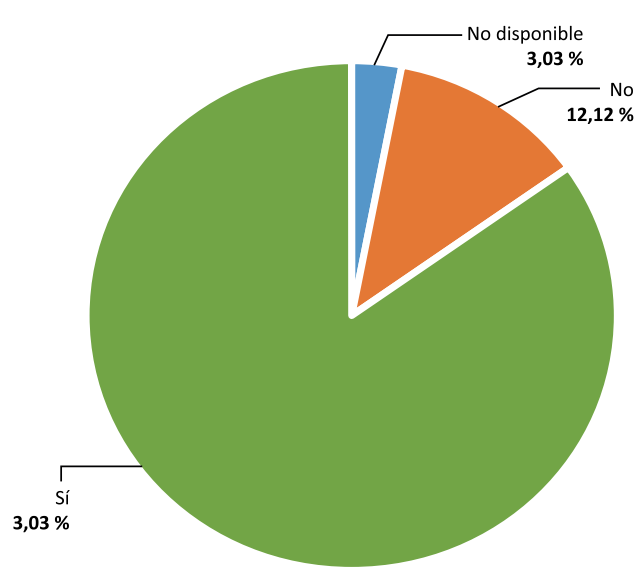

Figura 7

Consulta a la guía de prácticas

clínicas por el personal de la

E.S.E.

\section{CONCLUSIONES}

En el ejercicio de la exploración de la adherencia de los actores del ámbito de salud en la ciudad de Barranquilla, mediante el análisis realizado en la muestra seleccionada, se pudo evidenciar el estado de la categorización y calificación, del estado del conocimiento sobre la adherencia a las guías prácticas clínicas en las E.S.E. del Estado.

Los resultados finales permiten enunciar que el estado de adherencia actual se encuentra en un nivel alto que se proyecta en auge con referencia a 
todo el personal involucrado en la atención de la madre gestante con trastornos hipertensivos. Solo un 10 \% de la población indagada desconoce la existencia y alcance total de las guías de prácticas médicas, y un porcentaje cercano al $6 \%$ no supo responder a este interrogante. Como lo ha expuesto Bertoglia et al (2010), la cultura y dinamización del conocimiento en el campo médico es una labor constante que debe alimentarse sin cansancio. Lo anterior cobra pertinencia en el caso de estudio, ya que al promover el aprendizaje constante por parte de todos los actores médicos, los resultados pueden ser tanto óptimos como oportunos.

Otro aspecto de resaltar es la de la consulta a la guía: 84 \% viene realizando retroalimentación con el compendio, lo que brinda garantía y tranquilidad al interior de las IPS, ya que como lo ha expresado Gaillard et al (2011), en la medida que las herramientas generadas por los entes de investigación gubernamental o no gubernamental, sean consultadas, hay más posibilidades de acierto en cada uno de los diferentes casos que se pueden presentar, especialmente, en sucesos de urgencia con madres gestantes.

Los hallazgos en términos generales dejan un positivo balance en cuanto a la difusión y conocimiento de la guía de prácticas clínicas; sin embargo, es conveniente continuar con los programas de promoción y lograr la captura de atención de los profesionales, ya que aunque la guía está a disposición en momentos cruciales, las decisiones se vienen tomando con base en conceptos de colegas, consulta con otras fuentes o revisiones de casos anteriores.

Finalmente, se puede concluir que la importancia de los programas de promoción al interior del sector salud, especialmente las E.S.E. del Estado, son cruciales, no solo es necesario contar con el material de consulta, sino además, estimular que todas las herramientas e instrumentos diseñados 
para su aplicación sean tomados en cuenta, entre otros aspectos, porque una adecuada decisión puede salvar vidas e impactar de manera directa en las estadísticas negativas que quieren erradicarse en este campo.

\section{REFERENCIAS BIBLIOGRÁFICAS}

Arrieta, N., Blanquiceth, R., Borrero, E. \& Muños, V. (2015). Impacto del Programa de Hipertensión Arterial en un Centro de Salud de Soledad, Atlántico (Col). , Universidad Simón Bolívar, Revista Ciencia e Innovación en Salud 3(1), 27-32.

Bertoglia, P., Rivas, A., Navarrete, P., Castro, M., Acurio, J. \& Escudero, C. (2010). Resultados clínicos y perinatales de los embarazos con hipertensión arterial en un hospital de referencia de la VIII región de Chile. Rev Chil Obstet Ginecol, 75, 162-171.

Gaillard, R., Bakker, R., Willemsen, P., Hofman, A., Steegers, E., Jaddoe, W. (2011). Blood pressure tracking during pregnancy and the risk of gestational hypertensive disorders. The Generation $R$ Study. Eur Heart $J ., 32,3088-3097$.

Kovanci, E. \& Gregg, A. (2010). Blood Pressure Regulation Across Pregnancy: Evidence of a Paradigm Shift in Gene Expression. Hypertens Pregnancy, 29(2), 236-247.

Landázuri, P., Restrepo, B. \& Trejos, J. (2006). Perfil lipídico por trimestres de gestaciones en una población de mujeres colombianas. Rev Colomb Obstet Ginecol, 57, 256-263.

LilDommar, A., Suárez, C., Rojas G, Marcano, M. \& Nuccio, J. (2009). Hipertension Arterial Inducida por embarazo: Factores de riesgo asociados. Revista Saber, 21, 34-39.

MacDonald, C., Lawlor, A., Fraser, A., May, M., et al. (2012). Blood Pressure Change in Normotensive, Gestational Hypertensive, Preeclamptic, and Essential Hypertensive Pregnancies. Hypertension; 59:1241-1248. Ortega, J., Hernández, H., Mariano, H. \& Herrera, A. (2015). Calidad Es- 
tratégica en los Servicios de Salud. Centro de investigación y proyectos (CINPRO) Corporación Universitaria Latinoamericana. Barranquilla, Colombia.

Rodríguez, F., Martínez, J., KawaKarasik, S., Villanueva, L., Reyes, N., Flisser, A., et al. (2011). Comparison of hemodynamic, biochemical and hematological parameters of healthy pregnant women in the third trimester of pregnancy and the active labor phase. BMC Pregnancy Childbirth, 11, 11-33.

Romero, G., González B. (2011). Persistencia de hipertensión en mujeres con preeclampsia. Ginecol Obstet Mex, 79, 601-606.

Salas, A., Velázquez, E., Villarroel, V. \& Arata, G. (2004). Relación entre la concentración de leptina, insulina, norepinefrina y presión arterial en embarazadas sanas. Rev Venez Endocrinol Metab, 2, 10-14.

Thompson, M., Williams, M., Miller, R. (2009). Modelling the association of blood pressure during pregnancy with gestational age and body mass index. Paediatr Perinat Epidemiol, 23, 254-263.

Cómo citar este capítulo:

Beleño Agudelo, C., Salazar Araújo, M., Mercado Rodríguez, L., \& Altahona Rodríguez, Z. (2017). Atención de trastornos hipertensivos durante el embarazo en una empresa social del Estado de la ciudad de Barranquilla. En J. Rodríguez López, M. Gómez Barbosa, D. Martínez Sierra, H. Hernández Palma, C. Sierra García, C. Beleño Agudelo, ... J. Bermejo Urzola, Investigaciones y casos del sector salud de la región Caribe (pp.13-25). Barranquilla: Ediciones Universidad Simón Bolívar. 